\title{
Comment
}

\section{Legal Repercussions in Prescribing Benzodiazepines}

\author{
Peter Tyrer, Consultant Psychiatrist, Mapperley Hospital, Nottingham, NG3 6AA.
}

There is increasing concern over the medico-legal aspects of psychiatry and this is exemplified by Dr Spencer's suggestion that a special section of the College be devoted to these matters. ${ }^{1}$ Because of great public concern about the prescription and consumption of benzodiazepines, it is not surprising that caution in their prescription is being counselled for legal reasons as well as medical ones. In a recent paper ${ }^{2}$ Dr Ashton has outlined the now well-known problems that can arise from benzodiazepine prescription and also suggests that "a ground swell of litigation" is developing as "increasing numbers of patients apply for legal aid to take proceedings against drug companies marketing benzodiazepines and doctors prescribing them long-term".

Psychiatrists need to be aware of these developments if they are to avoid such proceedings being taken against them. When a medical practitioner (Dr Vernon Coleman) exhorts patients to sue doctors for prescribing benzodiazepines, the issue should not be dismissed lightly.

There are several areas of legal conflict, of which three are common. These concern the effects of benzodiazepines in causing accidents because of their psychomotor effects, both alone and in combination with other drugs (particularly alcohol), aggressive acts carried out while the patient is taking a benzodiazepine, and the suffering caused by longterm dependence and withdrawal reactions. The first two are related to the well-known effects of benzodiazepines on cognitive and memory function. Benzodiazepines lead to psychomotor impairment and induce anterograde amnesia, particularly in higher dosage. These effects are increased when another central depressant drug is taken. It is also well known that benzodiazepines can 'release' aggression in predisposed individuals and thus could be blamed at least to some extent when aggressive acts are committed.

Until recently most psychiatrists called upon to appear in court in cases such as these have not been blamed for their prescriptions of benzodiazepines. However, with increasing criticism of their long-term use, all doctors who prescribe them regularly for several months or longer are likely to come under criticism unless they can give a good reason for long-term prescribing. Paradoxically, those who have been prescribed benzodiazepines for many years have less of a case than those who have only taken them for five years or less. This is because it is only in recent years that the profession has become aware that pharmacological dependence can take place when benzodiazepines are prescribed long-term in therapeutic dosage. In the 1960s and 1970s this was not known and practitioners were being encouraged to prescribe benzodiazepines in place of the more dangerous barbiturates because they were safer and had a much lower risk of dependence.
There is no doubt that the suffering created in a patient who is genuinely dependent on benzodiazepines can be considerable and if negligence can be proved the damages could well be substantial. However, in many cases the pressure for continued prescription has come from patients and the alternatives to prescribing benzodiazepines might have been more dangerous (e.g. inappropriate use of alcohol) and there may often be considerable doubt about negligence in an individual patient. However, if a patient has been dependent on benzodiazepines and been withdrawn successfully it is important for the practitioner to be aware of the risks of represcribing a benzodiazepine. The doctor needs to establish if the patient has been dependent on benzodiazepines previously and would be unwise to represcribe without the patient's full knowledge and agreement of the risks involved. This also emphasises the importance of keeping adequate notes of consultations when benzodiazepines are being prescribed.

A more murky area is the difference in dependence potential between different benzodiazepines. There is increasing concern about the possibilities that benzodiazepines of higher potency and shorter half life may be at greater risk of producing dependence. Triazolam, lorazepam and alprazolam appear to be particularly at risk. Although much of the evidence for this is circumstantial and anecdotal it has recently become more substantial ${ }^{3-5}$ and can no longer be ignored in a court of law. The time has not yet come when a doctor could be successfully prosecuted for prescribing one of these benzodiazepines in preference to another but this is not an unimaginable scenario in the future.

Dr Ashton's article is a timely reminder that the prescription of benzodiazepines is now a high profile activity for the psychiatrist that has been scrutinised much more closely than many of our other functions. It is well to be aware that one of the potential onlookers is a lawyer.

\section{REFERENCES}

'SPENCER, D. A. (1987) The legal aspects of psychiatry. Bulletin of the Royal College of Psychiatrists, 11, 206-207.

${ }^{2}$ AsHTON, C. H. (1987) Dangers in medico-legal aspects of benzodiazepines. Journal of the Medical Defence Union, Summer 1987, 5-8.

${ }^{3}$ TYRER, P., RUTHERPoRd, D. \& HUGGet, T. (1981) Benzodiazepine withdrawal symptoms and propranolol. Lancet, 1, 520-522.

4Griffiths, R. R., Lamb, R. J., Ator, N. A., RoAChE, J. D. \& BraDY, J. V. (1985) The relative abuse liability of triazolam; experimental assessment in animals and humans. Neuroscience and Biobehavioural Reviews, 9, 133-151.

'FYeR, A. J., LIEBOWITZ, M. R., Gorman, J. M. et al (1987) Discontinuation of alprazolam treatment in panic patients. American Journal of Psychiatry, 144, 303-308. 